\title{
Enhancing the optoelectronic properties of amorphous zinc tin oxide by subgap defect passivation: A theoretical and experimental demonstration
}

\author{
Esteban Rucavado, ${ }^{1}$ Quentin Jeangros,,${ }^{1,2}$ Daniel F. Urban, ${ }^{3}$ Jakub Holovský, ${ }^{4,5}$ Zdenek Remes, ${ }^{4,5}$ Martial Duchamp, ${ }^{6}$ \\ Federica Landucci, ${ }^{1,7}$ Rafal E. Dunin-Borkowski, ${ }^{6}$ Wolfgang Körner, ${ }^{3}$ Christian Elsässer, ${ }^{3,8}$ Aïcha Hessler-Wyser, ${ }^{1}$ \\ Monica Morales-Masis, ${ }^{1}$ and Christophe Ballif ${ }^{1}$ \\ ${ }^{1}$ École Polytechnique Fédérale de Lausanne (EPFL), Institute of Microengineering (IMT), Photovoltaics and Thin Film Electronics \\ Laboratory, Rue de la Maladière 71b, CH-2002 Neuchâtel, Switzerland \\ ${ }^{2}$ University of Basel, Department of Physics, Klingelbergstrasse 82, CH-4056 Basel, Switzerland \\ ${ }^{3}$ Fraunhofer Institute for Mechanics of Materials (IWM), Wöhlerstraße 11, D-79108 Freiburg, Germany \\ ${ }^{4}$ Czech Technical University (CTU), Faculty of Electrical Engineering, Technická 2, 16627 Prague, Czech Republic \\ ${ }^{5}$ Institute of Physics of the Czech Academy of Sciences v. v. i., Cukrovarnická 10, 16200 Prague, Czech Republic \\ ${ }^{6}$ Forschungszentrum Jülich, Ernst Ruska-Centre for Microscopy and Spectroscopy with Electrons and Peter Grünberg Institute, \\ 52425 Jülich, Germany \\ ${ }^{7}$ École Polytechnique Fédérale de Lausanne (EPFL), School of Basic Sciences (SB), Interdisciplinary Centre for Electron Microscopy, \\ CH-1015 Lausanne, Switzerland \\ ${ }^{8}$ University of Freiburg, Freiburg Materials Research Center, Stefan-Meier-Straße 21, 79104 Freiburg, Germany
}

(Received 22 February 2017; revised manuscript received 28 April 2017; published 9 June 2017)

\begin{abstract}
The link between sub-bandgap states and optoelectronic properties is investigated for amorphous zinc tin oxide (a-ZTO) thin films deposited by RF sputtering. a-ZTO samples were annealed up to $500^{\circ} \mathrm{C}$ in oxidizing, neutral, and reducing atmospheres before characterizing their structural and optoelectronic properties by photothermal deflection spectroscopy, near-infrared-visible UV spectrophotometry, Hall effect, Rutherford backscattering, hydrogen forward scattering and transmission electron microscopy. By combining the experimental results with density functional theory calculations, oxygen deficiencies and resulting metal atoms clusters are identified as the source of subgap states, some of which act as electron donors but also as free electron scattering centers. The role of hydrogen on the optoelectronic properties is also discussed. Based on this detailed understanding of the different point defects present in a-ZTO, their impact on optoelectronic properties, and how they can be suppressed by postdeposition annealing treatments, an amorphous indium-free transparent conductive oxide, with a high thermal stability and an electron mobility up to $35 \mathrm{~cm}^{2} \mathrm{~V}^{-1} \mathrm{~s}^{-1}$, is demonstrated by defect passivation.
\end{abstract}

DOI: 10.1103/PhysRevB.95.245204

\section{INTRODUCTION}

Defects are ubiquitous and unavoidable in semiconductor materials. In amorphous semiconductors, dangling bonds created by undercoordinated atoms or atom deficiencies create subgap defect states [1]. Moreover, structural disorder in amorphous semiconductors causes exponential tails in the band-gap edge, which acts as a trap state for free electrons and holes [2]. For transparent conductive oxides (TCOs), point defects play a dual role; they can act as a source of free electrons, i.e. as dopants, shifting the Fermi level deep in the conduction band, and act as point defects that limit the free carrier mobility and might produce subgap states, which are detrimental to optical properties [3-7]. A fine tuning of these defects is hence required to adjust the optoelectronic properties of TCOs depending on the desired application. In that regard, several research groups have used density functional theory (DFT) calculations to assess the structural origin of electronic states inside the band gap. Körner et al. [8-11] developed a generic theory to explain the structural origin of subgap states in TCOs. It was demonstrated that uncoordinated oxygen generates deep states near the valence band, while oxygen deficiencies (or $\mathrm{V}_{\ddot{\mathrm{O}}}$ following the Kröger-Vink notation [12]) and resulting metal atom clusters create shallow donor states near the conduction band edge. Experimentally, Jayaraj et al. demonstrated the interplay between oxygen concentration and localized subgap donors in pulsed-laser-deposited amorphous zinc tin oxide ( $\mathrm{Zn}: \mathrm{Sn}=1: 1$ and 2:1) [13]. Both low and high oxygen concentrations were found to result in low free carrier concentration, as "free" electrons were numerous but trapped in localized states or low in number due to the small amount of donor states, respectively. Alternatively, Kamiya et al. [14,15] combined optical absorption measurements and DFT calculations to suggest that $\mathrm{V}_{\ddot{\mathrm{O}}}$ in amorphous $\mathrm{InGaZnO}_{2}$ may induce deep levels that could function as electron traps, instead of shallow donor states. In addition, they found that annealing in oxygen-rich atmospheres improved the electrical properties of the TCO by increasing the free carrier concentration, an effect that was attributed to a reduction of localized subgap electron traps. Alternatively, other studies have demonstrated that residual hydrogen, which may be trapped in the film during sputtering, should also be taken into account when investigating subgap states [16-21]. Van de Walle and co-workers used DFT with the local density approximation and generalized gradient approximation (LDA and GGA, respectively) to suggest that hydrogen, rather than $\mathrm{V}_{\ddot{\mathrm{O}}}$, is the main source of free carriers by acting as a shallow donor in $\mathrm{In}_{2} \mathrm{O}_{3}, \mathrm{SnO}_{2}$, and $\mathrm{ZnO}[17,22,23]$, in agreement with other reported experimental data [16,24-26].

Among the numerous TCO chemical compositions reported in literature [27,28], amorphous zinc tin oxide has the following advantages: it is indium-free and hence low cost, and at a $\mathrm{Zn} /(\mathrm{Zn}+\mathrm{Sn})$ composition of 0.1 has a stable microstructure at high temperature [29-31], presenting a total 
transmittance higher than $70 \%$ and an absorptance lower than $5 \%$ in the visible and near infrared [32-34], which makes it more transparent than commercially used indium tin oxide. Due to its amorphous microstructure, low surface roughness [30], and low free carrier density (i.e., restricted conductivity), it has been mainly applied in thin-film transistors as an active channel [35]. Zhu et al. [31] examined amorphous zinc tin oxide thin films (of composition $\mathrm{Zn} / \mathrm{Zn}+\mathrm{Sn}=0.1$ and 0.3 ) by synchrotron $x$-ray scattering and $x$-ray absorption spectroscopy and found that the presence of zinc atoms induces strain in the atomic network, which enhances the thermal stability of a-ZTO and delays its crystallization. This high thermal stability was exploited for the application of a-ZTO as a recombination layer in mesoscopic perovskite/silicon tandem solar cells, which requires processing temperatures up to $500{ }^{\circ} \mathrm{C}$ [36]. Yet questions remain about the actual crystallography of the films after annealing at temperatures above $400^{\circ} \mathrm{C}$, the effect of hydrogen during annealing, as well as the source of free carriers in a-ZTO [5,9,10,22,37,38].

It was recently demonstrated that a-ZTO with the specific composition of $\mathrm{Zn}_{0.05} \mathrm{Sn}_{0.30} \mathrm{O}_{0.65}$ (from here on called aZTO for simplicity), could be successfully employed as a transparent electrode in flexible OLEDs [35,39]. To further improve the optical and electrical properties of a-ZTO, the nature of the subgap states is investigated here in detail, as well as their effect on the optoelectronic properties. Based on electrical, optical, microstructural, chemical characterization, and DFT calculations, the role of point defects is assessed. Using this knowledge, the optoelectronic properties of the films are further enhanced.

\section{EXPERIMENTS}

a-ZTO was deposited on aluminoborosilicate glass using an Evatec Clusterline physical vapor deposition system. $150 \mathrm{~nm}$ thick films were deposited using a magnetron rf sputtering source using a power density of $0.32 \mathrm{~W} \mathrm{~cm}^{-2}$ and a substrate temperature of $60^{\circ} \mathrm{C}$ in an atmosphere of argon (Ar) and oxygen $\left(\mathrm{O}_{2}\right)$ with an $\mathrm{Ar} / \mathrm{O}_{2}$ flow ratio of 10/2. More details on the deposition parameters and the development of a-ZTO with this particular stoichiometry are described in Ref. [35]. The films were systematically annealed from $150{ }^{\circ} \mathrm{C}$ to $500^{\circ} \mathrm{C}$ in air, nitrogen, and hydrogen atmospheres separately. Annealing experiments in air were performed at atmospheric pressure, while nitrogen and hydrogen treatments were done at a pressure of 0.5 mbar using a constant gas flow. The thermal treatments were performed by heating the material for $30 \mathrm{~min}$ from room temperature to the annealing temperature to avoid high thermal stress on the substrate. The a-ZTO samples were then kept for $30 \mathrm{~min}$ at the chosen temperature, before being cooled down passively to room temperature.

The mobility $(\mu)$ and free carrier concentration $\left(N_{e}\right)$ of as-deposited and annealed films were obtained by Hall effect measurements in the Van der Pauw configuration using a HMS-5000 equipment. Temperature-dependent Hall effect measurements were performed from $-190^{\circ} \mathrm{C}$ to $45^{\circ} \mathrm{C}$ using the same tool. The optical total transmittance $(T T)$ and total reflectance $(T R)$ of a-ZTO were determined using UV-vis near-infrared (NIR) spectrophotometry (Lambda-950 equipment from Perkin Elmer). The absorptance $(A)$ was calculated from $A=100-T T-T R$. Photothermal deflection spectroscopy (PDS) was used to measure the absorptance of a-ZTO layers on fused silica. In this technique, the films are submerged in a temperature-sensitive liquid (Fluorinert FC-72) and illuminated by monochromatic light. Temperature gradients in the liquid, caused by light absorption in the film, induce a deflection of a probe laser. The direct relation between the laser deflection and the absorption of the films allows PDS to have higher sensitivity than spectrophotometry measurements. The details of PDS are described extensively by Boccara et al. [40]. This technique was performed using an in-house equipment based on a $150-\mathrm{W}$ xenon lamp. The absorption coefficient was measured in the energy range of $0.7-4.96 \mathrm{eV}$.

The microstructure and crystallography of as-deposited and annealed a-ZTO was investigated using transmission electron microscopy (TEM). TEM 50-nm-thick cross-sectional samples were prepared using the conventional focused ion beam lift-out technique in a Zeiss Nvision 40. As standard selected area diffraction patterns may fail to identify nanometric crystallites in an amorphous matrix, energy-filtered electron diffraction patterns were acquired in nanobeam conditions by scanning an electron probe in a raster over the thin sample in an FEI Titan S microscope operated at $300 \mathrm{kV}$. Information about short-range order (i.e., the first two coordination shells) in a-ZTO was obtained from radial profiles of diffraction patterns. The presence of medium-range order (from the third coordination shell to about $3 \mathrm{~nm}$ ) in a-ZTO was assessed using fluctuation electron microscopy [41] by computing the normalized variance of 1200 diffraction radial profiles per sample and per probe size $(1.5 \mathrm{~nm}$ for a convergence semiangle of $0.5 \mathrm{mrad}, 2 \mathrm{~nm}$ using $0.3 \mathrm{mrad}$, and $3 \mathrm{~nm}$ for $0.2 \mathrm{mrad}$ ). See Supplemental Material [42] for experimental details.

Rutherford backscattering (RBS) was done in a 6-MV HVEC Tandem accelerator using 2-MeV helium ions. Substrate signal and background arising from pileup events were subtracted. Hydrogen forward scattering (HFS) was performed by EAG Laboratories. These measurements were recorded using a detector $30^{\circ}$ from the forward trajectory of an incident $\mathrm{He}^{2+}$ ion beam. The incident beam strikes the surface with an angle of $75^{\circ}$ from the normal of the sample. Hydrogen concentrations were determined by comparing the number of hydrogen counts obtained from reference samples after normalizing the data by the stopping powers of the different materials.

\section{COMPUTATIONAL METHOD}

The experimental investigations outlined above were supported by atomistic first-principles simulations based on density functional theory. In order to achieve accurate and reliable theoretical results for electronic densities of states (DOS), a self-interaction correction (SIC) to the local density approximation was employed, which has recently been proven to be well suitable for the calculation of electronic defect levels in band gaps of the $\mathrm{Zn}-\mathrm{Sn}-\mathrm{O}$ system $[8,9]$.

\section{A. Generation of atomistic supercell models for a-ZTO}

Amorphous structures were generated by performing classical molecular dynamics (MD) with the GULP code [43] 
TABLE I. Compilation of the 38 supercell models generated by atomistic simulations, listing the number of samples ( $\left.n_{\text {samp }}\right)$, the supercell composition, the total number of atoms per supercell, and the atomic percentage of elements $\left(p_{x}\right)$.

\begin{tabular}{|c|c|c|c|c|c|c|c|}
\hline Structure & $n_{\text {samp }}$ & Supercell (no. of atoms) & $N_{\text {at }}$ & $p_{\text {Zn }}[$ at $\%]$ & $p_{\text {Sn }}[$ at $\%]$ & $p_{\mathrm{O}}[$ at $\%]$ & $p_{\mathrm{Zn}}[$ at $\%]$ \\
\hline ZTO "stoichiometric" & 8 & $\mathrm{Zn}_{4} \mathrm{Sn}_{27} \mathrm{O}_{58}$ & 89 & 4.50 & 30.3 & 65.2 & 0 \\
\hline ZTO “oxygen poor" & 8 & $\mathrm{Zn}_{4} \mathrm{Sn}_{27} \mathrm{O}_{57}$ & 88 & 4.55 & 30.7 & 64.8 & 0 \\
\hline $\mathrm{ZTO}: \mathrm{V}_{\ddot{\mathrm{O}}}$ & 7 & $\mathrm{Zn}_{4} \mathrm{Sn}_{27} \mathrm{O}_{57}$ & 88 & 4.55 & 30.7 & 64.8 & 0 \\
\hline $\mathrm{ZTO}: \mathrm{V}_{\ddot{\mathrm{O}}}+\mathrm{H}$ & 8 & $\mathrm{Zn}_{4} \mathrm{Sn}_{27} \mathrm{O}_{57} \mathrm{H}$ & 89 & 4.50 & 30.3 & 64 & 1.12 \\
\hline $\mathrm{ZTO}+\mathrm{H}$ & 7 & $\mathrm{Zn}_{4} \mathrm{Sn}_{27} \mathrm{O}_{58} \mathrm{H}$ & 90 & 4.44 & 30.0 & 64.4 & 1.11 \\
\hline
\end{tabular}

and empirical Buckingham-type interatomic potentials. The heat-and-quench cycle was started at $5000 \mathrm{~K}$ and cooled down in steps of $10 \mathrm{~K}$ per 0.5 ps with time steps of 1 fs at constant temperature. Then the supercells were structurally optimized in volume, cell shape, and atomic positions by means of DFT calculations.

The relaxations of interatomic forces and residual stresses were carried out with the Broyden-Fletcher-Goldfarb-Shanno algorithm using the projected augmented-wave (PAW) method [44] as implemented in the VASP code [45,46]. The LDA was used for the exchange-correlation functional and PAW pseudopotentials were used to describe $\mathrm{Zn}(3 d, 4 s, 4 p)$, Sn $(4 d, 5 s, 5 p)$, and $\mathrm{O}(2 s, 2 p)$ as valence electrons.

For this study, we constructed a set of 38 amorphous structures, see Table I. Initially, two subsets of samples were generated. To ensure valid statistical results, eight samples with composition $\mathrm{Zn}_{4} \mathrm{Sn}_{27} \mathrm{O}_{58}$ were used to reflect the experimentally as-deposited ZTO films. These samples were labeled "stoichiometric," since their composition corresponds to an admixture of $4 \mathrm{ZnO}$ units and $27 \mathrm{SnO}_{2}$ units. The second subset of eight samples with the composition $\mathrm{Zn}_{4} \mathrm{Sn}_{27} \mathrm{O}_{57}$ is oxygen deficient and can be considered as systems with an annealed oxygen vacancy. From the stoichiometric set, we derived three further sets of samples by adding/removing some atoms and subsequently repeating the DFT procedure of structural optimization outlined above. (i) By removing an oxygen atom, we generated a local oxygen deficiency resembling an oxygen vacancy of a crystal (ZTO: $\mathrm{V}_{\ddot{\mathrm{O}}}$ ). (ii) Hydrogen-rich supercells were generated by adding a hydrogen atom $(\mathrm{ZTO}+\mathrm{H})$. (iii) Hydrogen atoms were added close to the oxygen deficiency sites of (i) to study the combined effect of $\mathrm{V}_{\ddot{\mathrm{O}}}$ and $\mathrm{H}$ on the DOS (ZTO : $\left.\mathrm{V}_{\ddot{\mathrm{O}}}+\mathrm{H}\right)$.

In the ZTO: $\mathrm{V}_{\ddot{\mathrm{O}}}$ group of structures, the removal of the oxygen atom is done after the MD amorphization. In contrast, the structures of the "oxygen-poor" system are relaxed to an energy minimum already with the reduced oxygen concentration.

\section{B. Electronic-structure calculations}

The electronic-structure calculations on the basis of the SIC-LDA were performed using the computational mixedbasis pseudopotential (MBPP) method [47-50]. For Zn, Sn, and $\mathrm{O}$, optimally smooth norm-conserving pseudopotentials were constructed and a mixed basis of plane waves and nonoverlapping localized orbitals were used. Due to the localized orbitals, a plane-wave cutoff energy of $20 \mathrm{Ry}$ $(1 \mathrm{Ry}=13.606 \mathrm{eV})$ is sufficient to obtain well-converged results. For the $k$-point sampling of the Brillouin-zone inte- grals, a Monkhorst-Pack mesh of $3 \times 3 \times 3$ and a Gaussian broadening of $0.2 \mathrm{eV}$ were used. The DOS of the supercells were evaluated with the same mesh and a Gaussian broadening of $0.1 \mathrm{eV}$.

The artificial self-interaction of the LDA is corrected by an incorporation of the SIC in the pseudopotentials [51,52]. The SIC procedure uses weight factors $\mathbf{w}=\left(\mathrm{w}^{(\mathrm{s})}, \mathrm{w}^{(\mathrm{p})}, \mathrm{w}^{(\mathrm{d})}\right)$ accounting for the occupations of the individual $s, p$, and $d$ valence orbitals. The $\mathrm{Zn} 3 d$ and $\mathrm{Sn} 4 d$ semicore orbitals were corrected by $100 \%$, i.e., $\mathbf{w}_{\mathrm{Zn}}=\mathbf{w}_{\mathrm{Sn}}=(0,0,1)$. The localized O- $2 s$ semicore orbitals are also corrected by $100 \%$, while for the spatially more extended O- $2 p$ valence-band orbitals $90 \%$ was considered, which implies the weight factors $\mathbf{w}_{\mathrm{O}}=(1,0.9,0)$. The latter choice is a compromise between the optimal values of $\mathrm{w}^{(\mathrm{p})} \mathrm{O}=0.8$ for $\mathrm{ZnO}$ and $\mathrm{w}^{(\mathrm{p})} \mathrm{O}=0.93$ for $\mathrm{SnO}_{2}$, which have been found to reproduce the band gaps of the binary crystalline compounds [9]. The main reason for the necessary adjustment of $\mathrm{w}^{(\mathrm{p})} \mathrm{O}$ is the change from the fourfold (tetrahedral) coordination of ions in wurtzite $\mathrm{ZnO}$ to the sixfold (octahedral) coordination of ions in rutile $\mathrm{SnO}_{2}$. The choice of $\mathrm{w}^{(\mathrm{p})} \mathrm{O}=0.9$ for the SIC of oxygen in the calculations of all ternary $\mathrm{Zn}-\mathrm{Sn}-\mathrm{O}$ compounds leads to an electronic band gap of $3.8 \mathrm{eV}$ for crystalline $\mathrm{ZnSnO}_{3}$, which is in very good agreement with experimental values [8]. For the adjustment of the atomic SIC to the crystal field in the solids, a scaling factor $\alpha=0.8$ is applied.

\section{EXPERIMENTAL RESULTS AND DISCUSSION}

\section{A. Electrical properties: Hall mobility and free carrier density}

The as-deposited 150 nm-thick a-ZTO films have an average $\mu$ of $21 \mathrm{~cm}^{2} \mathrm{~V}^{-1} \mathrm{~s}^{-1}$ and $N_{e}$ of $\sim 7 \times 10^{19} \mathrm{~cm}^{-3}$ (from a total of 20 samples). Two different behaviors are then observed upon annealing, depending on temperature (Fig. 1). At low temperature (low- $T$ in Fig. 1), i.e., $150{ }^{\circ} \mathrm{C}, 200^{\circ} \mathrm{C}$, and $300^{\circ} \mathrm{C}$, both $N_{e}$ and $\mu$ increase with annealing temperature regardless of the treatment atmosphere.

At higher temperatures (high- $T$, i.e., at $400^{\circ} \mathrm{C}$ and $500^{\circ} \mathrm{C}$ ), $N_{e}$ and $\mu$ are found to depend strongly on the annealing atmosphere. Thermal treatments in air result in an increase in $\mu$ from $20 \mathrm{~cm}^{2} \mathrm{~V}^{-1} \mathrm{~s}^{-1}$ up to $35 \mathrm{~cm}^{2} \mathrm{~V}^{-1} \mathrm{~s}^{-1}$, while $N_{e}$ slightly decreases from $7.0 \times 10^{19} \mathrm{~cm}^{-3}$ to $6.0 \times 10^{19} \mathrm{~cm}^{-3}$. Similar changes in conductivity with annealing in air have been reported for zinc tin oxide [29,31,53]. On the other hand, samples annealed in $\mathrm{N}_{2}$ in the high- $T$ range show a different behavior; $N_{e}$ increases up to $1.3 \times 10^{20} \mathrm{~cm}^{-3}$, while $\mu$ stabilizes at $26 \mathrm{~cm}^{2} \mathrm{~V}^{-1} \mathrm{~s}^{-1}$. Films treated in $\mathrm{H}_{2}$ at high temperatures exhibit the highest increase in $N_{e}$ (up to 


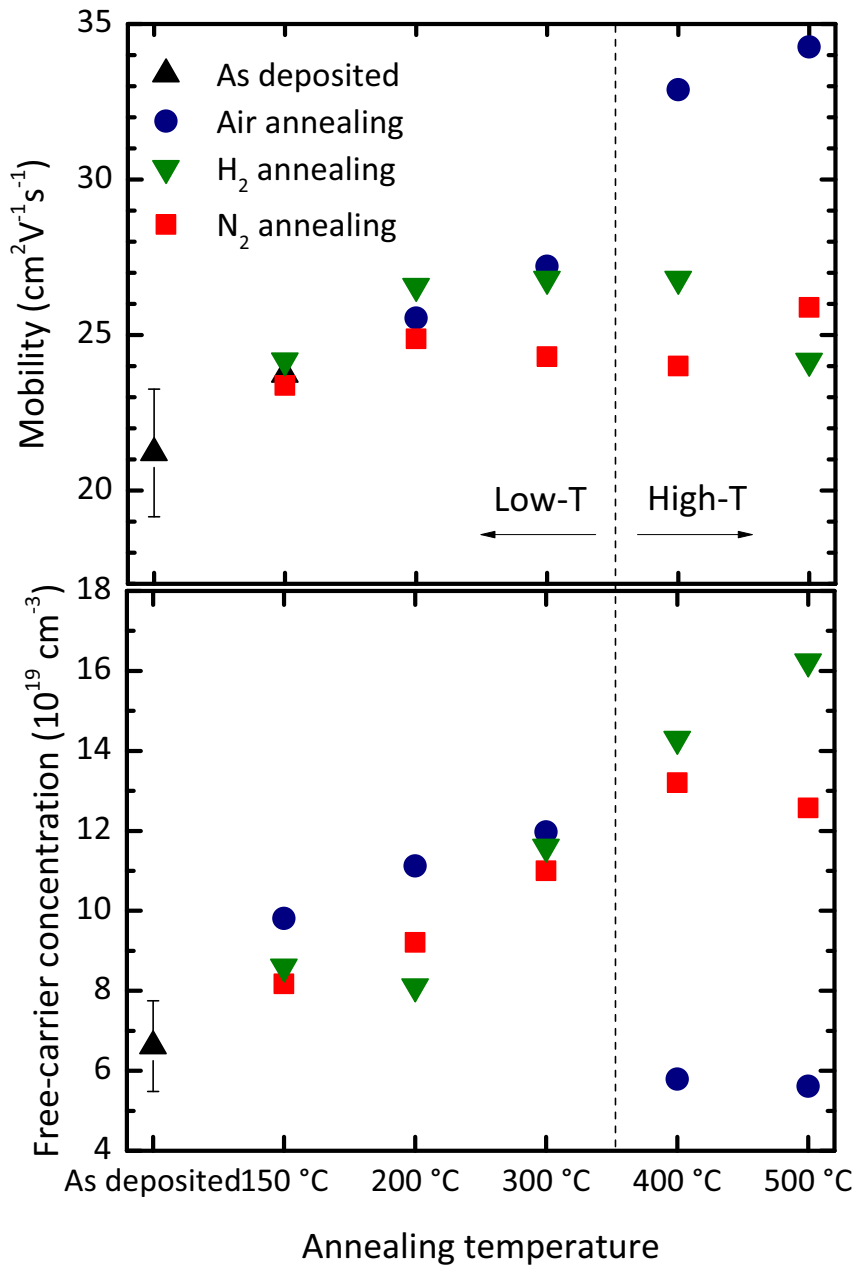

FIG. 1. Hall mobility $(\mu)$ and free carrier density $\left(N_{e}\right)$ of a-ZTO $\left(\mathrm{a}-\mathrm{Zn}_{0.05} \mathrm{Sn}_{0.30} \mathrm{O}_{0.65}\right)$ thin films as a function of annealing temperature. The dashed line divides the two different regimes observed at low and high temperatures.

$1.6 \times 10^{20} \mathrm{~cm}^{-3}$ when annealing at $500^{\circ} \mathrm{C}$ ). On the other hand, $\mu$ decreases after an initial increase at low temperature, and a final value of $24 \mathrm{~cm}^{2} \mathrm{~V}^{-1} \mathrm{~s}^{-1}$ is measured at $500^{\circ} \mathrm{C}$, which is slightly higher than the value obtained after deposition (of $21 \mathrm{~cm}^{2} \mathrm{~V}^{-1} \mathrm{~s}^{-1}$ ).

\section{B. Optical properties: Total transmittance and absorptance}

Figure 2 shows the total transmittance $T T$ and absorptance $A$ of samples either as-deposited, annealed, at $150^{\circ} \mathrm{C}$ or $500{ }^{\circ} \mathrm{C}$ in different atmospheres. As-deposited a-ZTO exhibits a TT higher than $80 \%$ and an $A$ down to $2 \%$ in the visible and infrared. Samples annealed at $150{ }^{\circ} \mathrm{C}$ change only slightly compared to the as-deposited a-ZTO, regardless of the annealing atmosphere [Fig. 2(a)]. The slight increase in absorptance in the near-IR region for samples annealed in air is assumed to be a consequence of free carrier absorption (FCA), caused by the modest increase in $N_{e}$ after such treatment.

On the other hand, clear differences are observed after annealing at $500{ }^{\circ} \mathrm{C}$ [Fig. 2(b)]. The films annealed at $500{ }^{\circ} \mathrm{C}$ in $\mathrm{H}_{2}$ absorb more in the NIR as a consequence of FCA, which can be described by the Drude model [54]. Alternatively,

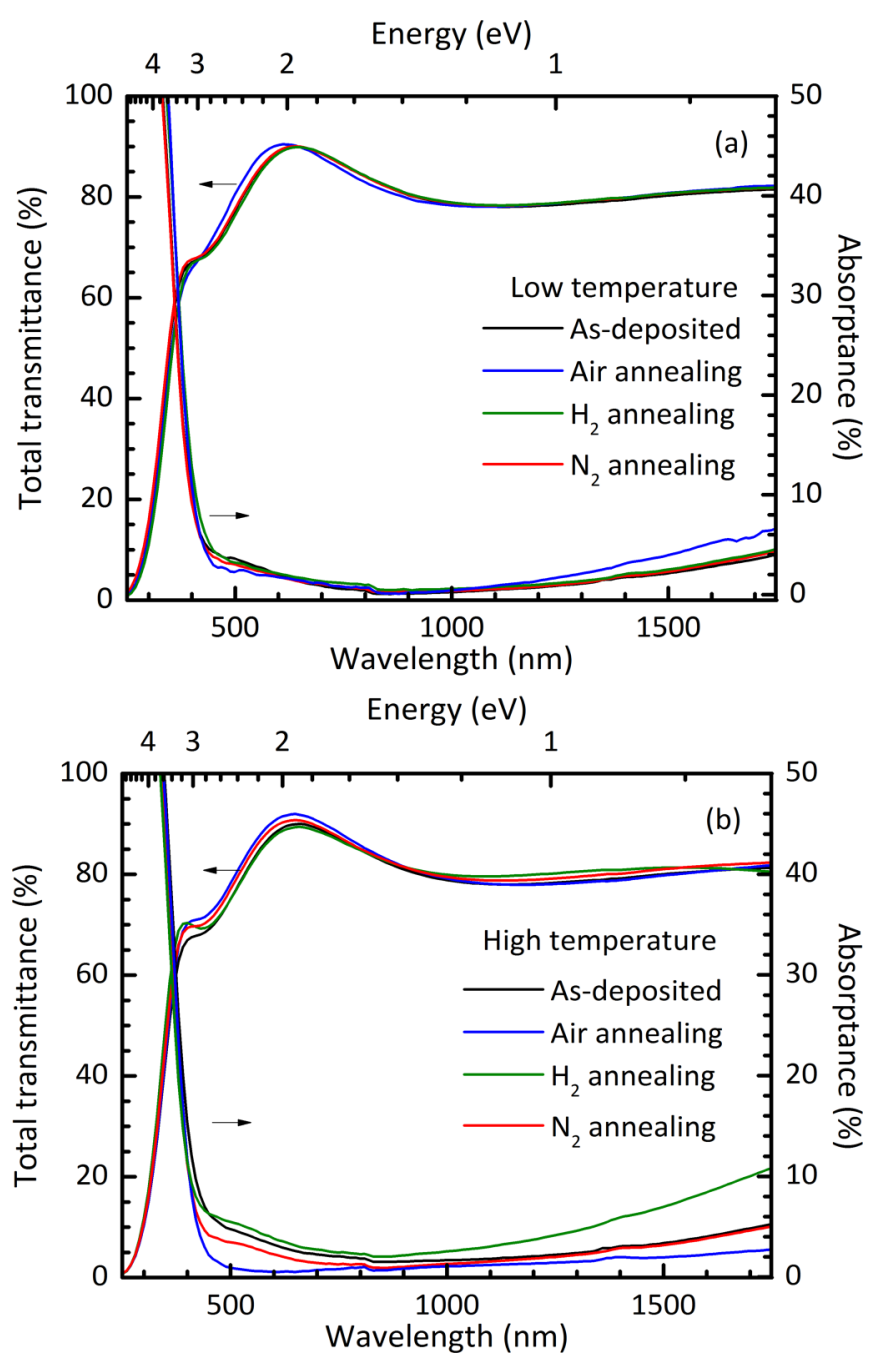

FIG. 2. Total transmittance and absorptance of a-ZTO asdeposited and annealed at $150{ }^{\circ} \mathrm{C}$ (a) and at $500^{\circ} \mathrm{C}$ (b) in air, nitrogen, and hydrogen.

samples annealed in air show considerably lower FCA since $N_{e}$ is lower in these films.

In the visible part of the spectrum, as-deposited films present a slightly increased absorption approaching the band edge (around $500 \mathrm{~nm}$ ). This could be detrimental to the use of a-ZTO in optoelectronic devices with high spectral sensitivity in the visible range. Only the samples treated in air at $500{ }^{\circ} \mathrm{C}$ do not exhibit this absorption center in the visible.

A more detailed analysis of these absorption centers was performed by PDS measurements [Fig. 3]. Figure 3(a) compares the absorption coefficient of as-deposited a-ZTO (black solid line), annealing in air at $150{ }^{\circ} \mathrm{C}$ (dashed blue) and at $500{ }^{\circ} \mathrm{C}$ (solid blue line). Films as-deposited and annealed at $150{ }^{\circ} \mathrm{C}$ show a broad sub-bandgap absorption at $2 \mathrm{eV}$, while samples treated in air at $500{ }^{\circ} \mathrm{C}$ exhibit a strong decrease in absorptance at this photon energy, confirming the observations of Fig. 2. Alternatively, the absorption coefficients of the samples annealed in $\mathrm{N}_{2}$ and $\mathrm{H}_{2}$ [Figs. 3(b) and 3(c)] change only slightly with temperature in this energy range. Figure 3(d) shows a comparison of films as-deposited and annealed at $500^{\circ} \mathrm{C}$ in all three atmospheres. Using the absorption 


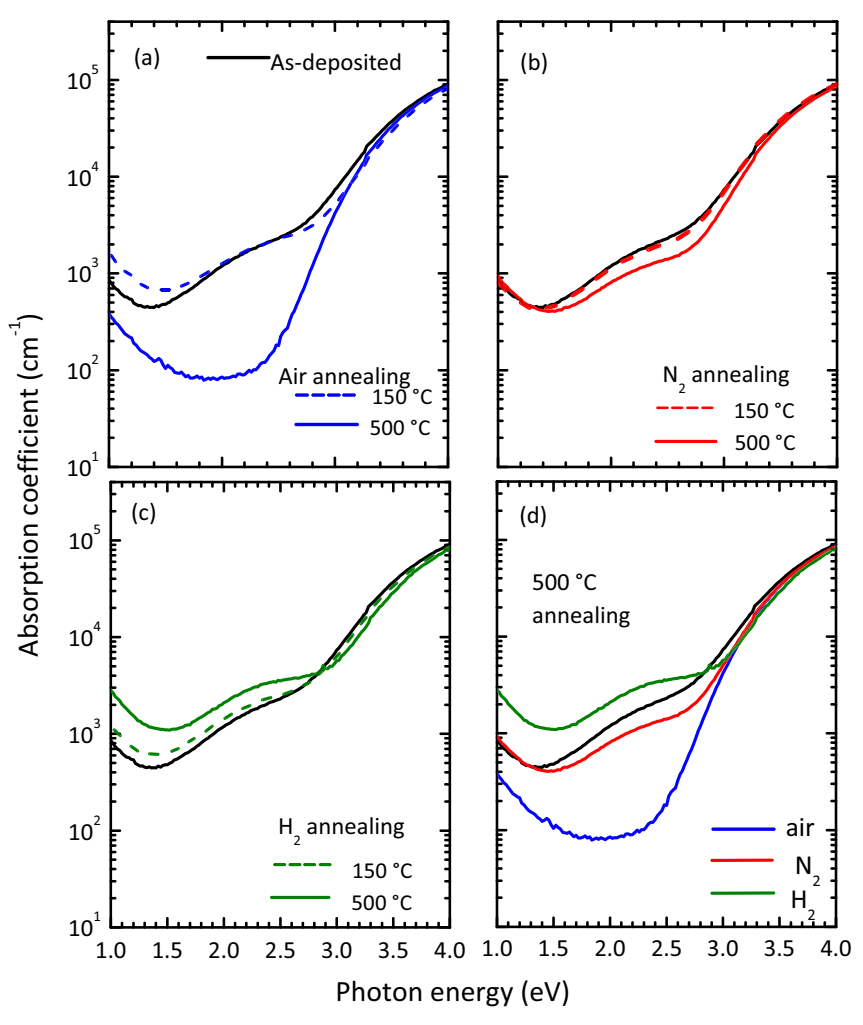

FIG. 3. Photothermal deflection spectroscopy of as-deposited aZTO (black line) and samples annealed in (a) air, (b) nitrogen, and (c) hydrogen at $150{ }^{\circ} \mathrm{C}$ (dashed colored lines) and $500^{\circ} \mathrm{C}$ (solid colored lines). A comparison between the films as-deposited and annealed at $500{ }^{\circ} \mathrm{C}$ is shown in (d) (blue for air, red for nitrogen, and green for hydrogen).

coefficient measured on the film annealed in air at $500^{\circ} \mathrm{C}$ and the Tauc equation [55], an optical band gap of $3.7 \mathrm{eV}$ is calculated, which is in agreement with literature reports $[32,56]$. It was not possible to measure the band gap of the as-deposited films since the subgap absorption interfered with the Tauc approach.

\section{Film composition and microstructure}

With respect to chemical composition, RBS measurements show that as-deposited and annealed films retain the atomic metallic molar fraction $\mathrm{Zn}_{0.05} \mathrm{Sn}_{0.3} \mathrm{O}_{\mathrm{x}}$, while the $\mathrm{O}$ content varies with the annealing atmosphere (Table II). After annealing at $T>500^{\circ} \mathrm{C}$ in air, the atomic concentration of oxygen increases by $1 \%$, while thermal treatments in $\mathrm{H}_{2}$ result in a decrease of $1 \%$ of the atomic ratio from the as-deposited material.

TABLE II. RBS results for as-deposited ZTO and ZTO annealed at $500{ }^{\circ} \mathrm{C}$ in different atmospheres.

\begin{tabular}{ll}
\hline \hline Material & Results from RBS \\
\hline ZTO as deposited & $\mathrm{Zn}_{0.049} \mathrm{Sn}_{0.299} \mathrm{O}_{0.652}$ \\
ZTO annealed in air & $\mathrm{Zn}_{0.047} \mathrm{Sn}_{0.289} \mathrm{O}_{0.664}$ \\
ZTO annealed in nitrogen & $\mathrm{Zn}_{0.049} \mathrm{Sn}_{0.304} \mathrm{O}_{0.652}$ \\
ZTO annealed in hydrogen & $\mathrm{Zn}_{0.051} \mathrm{Sn}_{0.308} \mathrm{O}_{0.641}$ \\
\hline \hline
\end{tabular}

This confirms the increase (decrease) of oxygen atoms in the amorphous network after annealing in oxygen (hydrogen)rich atmospheres, respectively. HFS measurements indicate that the as-deposited a-ZTO films contain around 0.7 at $\%$ of hydrogen. The high temperature annealing in air reduces the content of hydrogen to less than 0.5 at\% (under the detection limit of the HFS technique), while annealing in $\mathrm{H}_{2}$ at $500{ }^{\circ} \mathrm{C}$ results in an increase of hydrogen content up to 1.2 at $\%$. The increased amount of $\mathrm{H}$, together with the increase in $N_{e}$ observed in Fig. 1, suggests the role of hydrogen as a dopant $[16,19,57,58]$. In addition, $\mathrm{H}$ may also act as a reducing agent for oxygen [34], hence further increasing the $\mathrm{V}_{\ddot{\mathrm{O}}}$ density and in turn sub-band-gap absorption, as observed in Fig. 3.

The changes observed in optoelectronic properties discussed previously do not appear to be linked to a change in microstructure of the samples. Indeed, the nanobeam diffraction patterns acquired after deposition and after annealing at $500^{\circ} \mathrm{C}$ show hazy rings broken into speckles due to the small number of atoms in the probe [41]. This demonstrates the absence of nanometric crystallites in the samples before and after annealing at $500{ }^{\circ} \mathrm{C}$ in air [Figs. 4(c) and 4(d)]. Moreover, both short- and medium-range order do not appear to evolve significantly, as both the radial profiles shown in Figs. 4(e) and 4(f) and the normalized variances of radial profiles of hundreds of nanobeam diffraction patterns (see Supplemental Materials [42] for the variance of the diffraction patterns) appear insensitive to annealing under these conditions. These results support the conjecture that the generation, modification, and annihilation of point defects during the different annealing procedures are responsible for the observed changes in the optical and electrical properties of the a-ZTO films.

\section{Brooks-Herring-Dingle Model}

Temperature-dependent Hall effect measurements performed in the range from $-190^{\circ} \mathrm{C}$ to $45^{\circ} \mathrm{C}$ indicate that $N_{e}$ and $\mu$ of the as-deposited and annealed samples are independent of temperature (see Supplemental Materials [42]). This indicates that scattering by ionized and neutral impurities is the dominant mobility-limiting mechanism in all films assessed here [59-61].

Scattering of free carriers by ionized impurities is commonly described by the Brooks-Herring-Dingle (BHD) model [62]. This model considers the effect of a screened Coulomb potential on the relaxation time between two different scattering events that a charge carrier encounters, resulting in an equation for the mobility limited by ionized impurities, $\mu_{i i}$, as

$$
\mu_{i i}=\frac{3 \pi \hbar^{3}\left(4 \pi \varepsilon_{0} \kappa\right)^{2}}{2 Z^{2} e^{3} m^{* 2}} \times \frac{N_{e}}{N_{i}} \times \frac{1}{F(\xi)},
$$

with $F(\xi)=\ln (\xi+1)-\frac{\xi}{\xi+1}$ and $\xi=4 \pi^{3}\left(\frac{3}{\pi}\right)^{1 / 3} \frac{\varepsilon_{0} \kappa \hbar^{3} N_{e} \kappa^{1 / 3}}{e^{2} m^{*}}$, where $F(\xi)$ is the screening function, $\kappa$ the relative permittivity, $\varepsilon_{0}$ the vacuum permittivity, $N_{i}$ the concentration of ionized impurities, $Z$ the charge of the impurity, $m^{*}$ the effective mass, $e$ the elementary electron charge, and $\hbar$ the reduced Planck constant. To apply this model to the experimental data, $\mu$ vs $N_{e}$ was plotted in Fig. 5, including the data shown Fig. 1 and the result from the BHD model. 

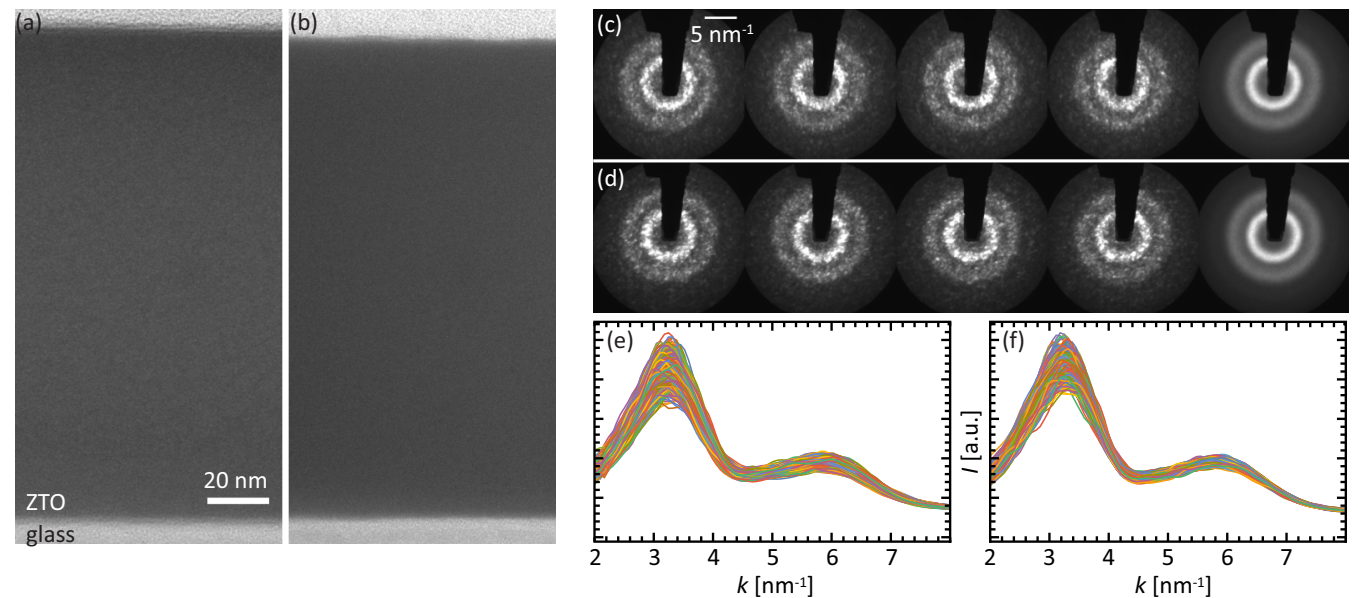

FIG. 4. TEM analysis of the samples after deposition and after annealing at $500{ }^{\circ} \mathrm{C}$ in air. (a, b) TEM images of focused ion beam-prepared cross sections after deposition and annealing at $500^{\circ} \mathrm{C}$, respectively. (c, d) Selection of nanobeam electron diffraction patterns of the samples shown in (a) and (b), respectively. The last inset of each series shows the mean of 100 nanobeam diffraction patterns. (e, f) Rotationally averaged diffracted intensities of 1200 nanobeam diffraction patterns of the samples shown in (a) and (b), respectively, highlighting the absence of both crystallinity and significant restructuring upon annealing. (See Supplemental Materials [42] for further details.)

The $m^{*}$ values were obtained from Fourier transform infrared spectroscopy reflectance measurements and Drude model fitting (see Supplemental Materials [42]). Effective masses of $0.30 m_{e}$ and $0.26 m_{e}$ were obtained for the films as-deposited and annealed at $500^{\circ} \mathrm{C}$ in air, respectively. Assuming that the majority of impurities in a-ZTO films have a charge $Z$ of +2 , the BHD model indicates that the $\mu-N_{e}$ data of the samples as-deposited and annealed at temperatures lower or equal to $300{ }^{\circ} \mathrm{C}$ in $\mathrm{H}_{2}$ and $\mathrm{N}_{2}$ atmosphere are described by the model, assuming a $N_{i}$ value

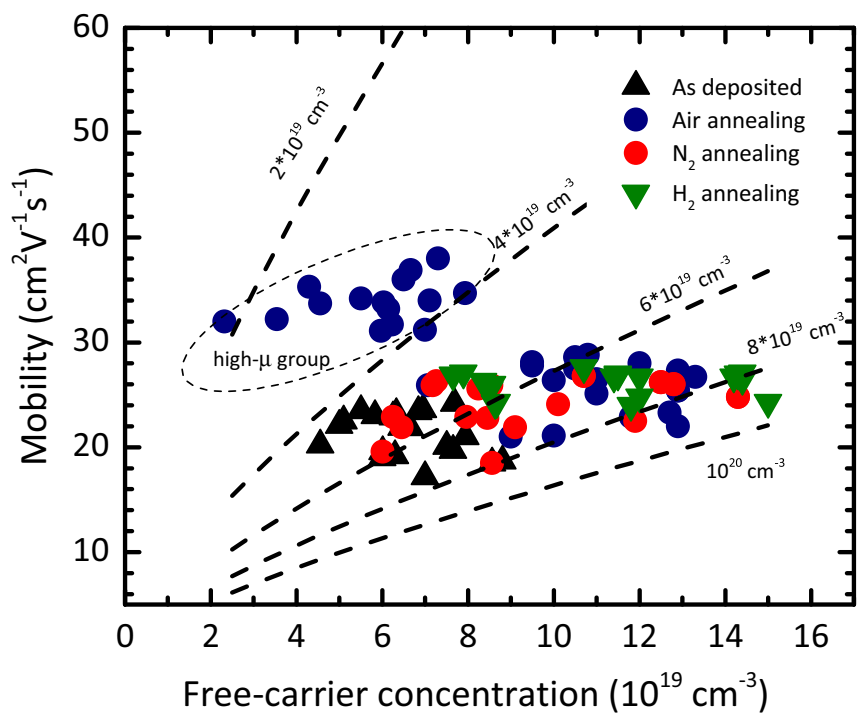

FIG. 5. Hall mobility plotted as function of free carrier density for films annealed in air, hydrogen, and nitrogen atmospheres. Lines are simulations using the Brooks-Herring-Dingle model, considering ionized defects of charge $Z=+2$ and of defect density $N_{i}$ changing from $2 \times 10^{19} \mathrm{~cm}^{-3}$ to $2 \times 10^{20} \mathrm{~cm}^{-3}$. Effective masses of $0.26 m_{e}$ and $0.3 m_{e}$ were used in the model, as calculated from Fourier transform infrared reflectance and Drude model fitting. of $\sim 5 \times 10^{19} \mathrm{~cm}^{-3}$ to $1 \times 10^{20} \mathrm{~cm}^{-3}$ [63]. The group of data with higher mobility, i.e., between 30 and $40 \mathrm{~cm}^{2} \mathrm{~V}^{-1} \mathrm{~s}^{-1}$ (annealed in air at $500^{\circ} \mathrm{C}$ ), are described by a much lower $N_{i}$, ranging from $2 \times 10^{19} \mathrm{~cm}^{-3}$ to $4 \times 10^{19} \mathrm{~cm}^{-3}$. Even though the experimental data does not the follow the trend set by the model when using constant $N_{i}$ values, these results suggest that annealing in air at temperatures above $300{ }^{\circ} \mathrm{C}$ reduces the density of ionized impurity defects, leading to the observed improvement in $\mu$ [Fig. 1] and to the clear reduction of subband-gap defects observed in the PDS measurements [Fig. 3].

\section{DFT CALCULATIONS}

The 38 amorphous sample structures defined in Table I were studied in terms of their electronic density of states. Figure 6(a) shows the DOS of all eight stoichiometric samples as calculated by our SIC-LDA approach. The curves were aligned with respect to the major peak in the valence band. The DOS of each sample shows characteristic tails attributed to the local disorder of bond lengths and angles, resulting in the overall effect of reducing the optical band gap. The valence band (VB) tail is much more pronounced than the conduction band (CB) tail. This can be explained by the direction dependence of the orbitals forming the respective bands. The cation spherical $s$ orbitals forming mainly the CB are rather insensitive to structural disorder, while the directiondependent $\mathrm{O} 2 p$ orbitals, which mainly form the upper part of the VB, are influenced strongly by structural disorder. This effect is well known for amorphous and polycrystalline semiconductors [64]. A quantitative analysis of the band tails of a-ZTO can be found in Ref. [9]. An enlarged view of the VB edge is shown in the inset of Fig. 6, where the curves are plotted on a logarithmic scale and the thick line represents the average of the eight curves.

Superimposed on the VB tail there are a variety of localized defect states whose distribution in position is highly sample dependent. Nevertheless, all these defect states can be attributed to undercoordinated oxygen atoms by means of an 

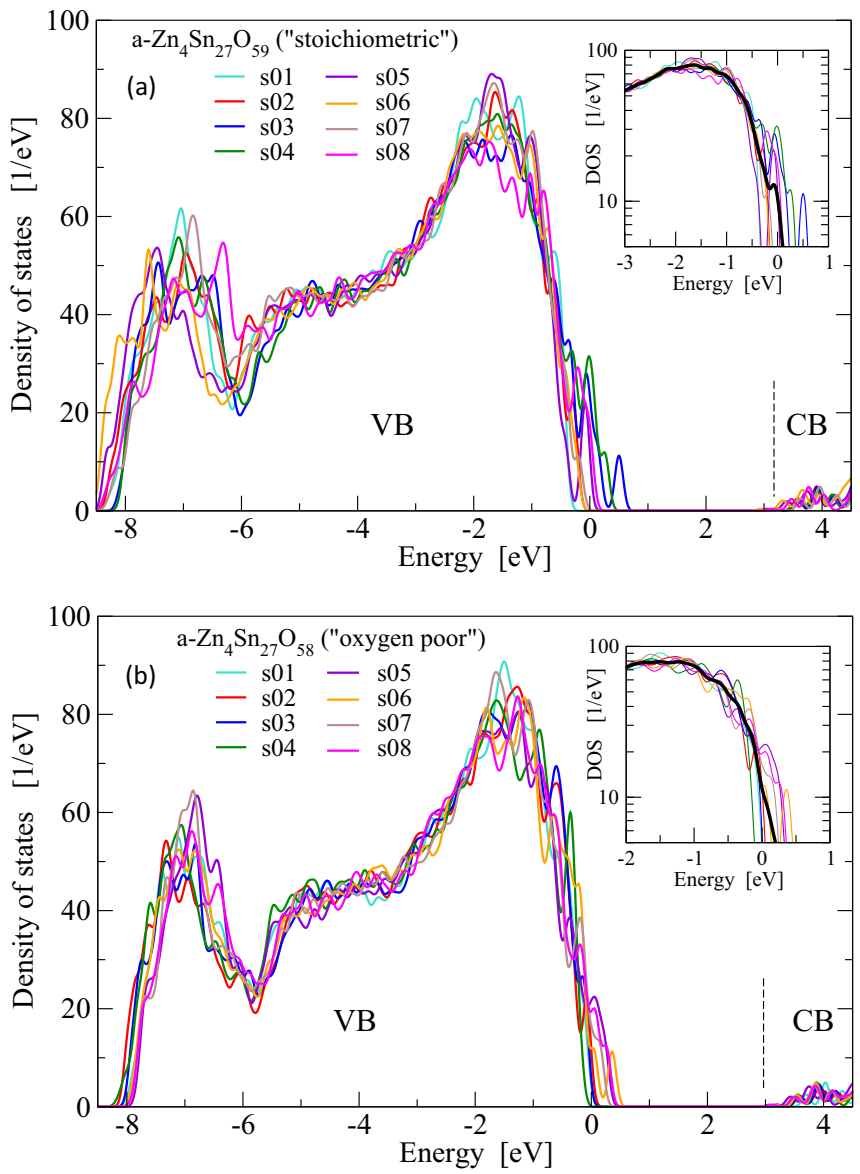

FIG. 6. Total densities of states of (a) eight stoichiometric and (b) eight oxygen-poor a-ZTO samples calculated by DFT with SIC-LDA. Superimposed on the VB tail are a number of localized defect states which can be attributed to undercoordinated oxygen atoms. The insets show the VB edge plotted on a log scale, while the thick lines show the average of the eight curves.

analysis of the atom-projected local densities of states (LDOS). These defects are less common if the oxygen content in the samples (prior to the MD amorphization) is reduced, since this would result in less undercoordinated oxygen.

The DOS of the eight annealed oxygen-poor samples (cf. Table I) are shown in Fig. 6(b) for comparison. These oxygendeficient samples do not show any oxygen vacancy-like defect states (which, if present, would be expected to be located in the vicinity of the $\mathrm{CB}$ ), since during the MD melt-and-quench procedure any of such local atomic configuration gets annealed completely.

The large Sn content in the films explains the effectiveness of the annealing, since this cation is rather flexible in its oxidation state and its respective atomic coordination, cf. the sixfold coordination in rutile $\mathrm{SnO}_{2}$, as opposed to the fourfold coordination in $\mathrm{SnO}$.

The picture changes qualitatively when considering ZTO: $V_{\ddot{O}}$ samples, which contain a local atomic environment similar to an oxygen vacancy in a crystal. This is referred to as a local oxygen deficiency in the amorphous sample. These structures are generated by removing an oxygen atom from the stoichiometric samples (followed by the DFT structural

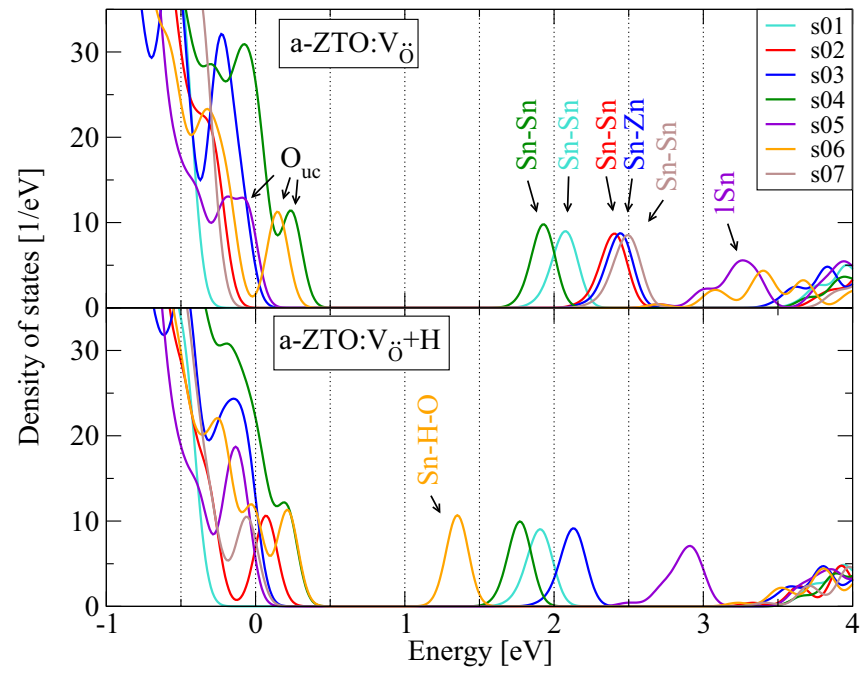

FIG. 7. Densities of states of (top panel) the a-ZTO: $\mathrm{V}_{\ddot{\mathrm{O}}}$ samples containing a local oxygen deficiency and (bottom panel) the a-ZTO : $\mathrm{V}_{\ddot{\mathrm{O}}}+\mathrm{H}$ samples with an additional $\mathrm{H}$ atom in the vicinity of the defect, calculated by DFT with SIC-LDA. The curves are aligned with respect to the main peak in the VB. Deep levels within the gap are mainly carried by one or two metal atoms (indicated in the top panel), while levels close to the VB stem from undercoordinated oxygen atoms $\left(\mathrm{O}_{\mathrm{uc}}\right)$. The defect state of the a-ZTO: $\mathrm{V}_{\ddot{\mathrm{O}}}+\mathrm{H}$ sample $\mathrm{s} 06$ is related to a $\mathrm{Sn}-\mathrm{H}$ bonding and neighboring $\mathrm{O}$ atoms.

optimization). The DOS of these samples are shown in Fig. 7(a). Distinct localized defect levels appear in the upper half of the band gap for the majority of samples. An analysis of atom-projected LDOS allows one to quantify the contributions of individual atoms to a specific defect level. With LDOS, it is also possible to calculate the weight factors by integration of the LDOS of the respective atoms in the relevant energy interval and subsequent division by the sum of the LDOS of all atoms integrated over the same energy range.

This LDOS analysis reveals that most of the weight of each of these levels is carried by only a few atoms, mostly two metal atoms and, to much less extent, one or two additional oxygen atoms located nearby. In recent studies on IGZO, ITO, and IZO, this type of metal-metal clustering was identified to be the common origin of the deep levels in the upper part of the band gap $[65,66]$. The same interpretation was recently shown to hold for amorphous $\mathrm{SnO}_{2}$ and $\mathrm{SnO}$ by Wahila et al. [67] using the Heyd-Scuseria-Ernzerhof (HSE) hybrid functional in their DFT calculations.

The specific position of these defect states within the upper half of the band gap in general shifts deeper into the band gap, the shorter the interatomic distance between the two metal atoms involved. Nevertheless, no one-to-one correlation between the energy and this distance can be established. Instead, the energetic positions of the metal-metal defect levels depend on the whole neighborhood (e.g., the number and distance of oxygen atoms that attract electrons from the metal atoms).

In order to study the effect of hydrogen in amorphous samples and on the deep levels, individual hydrogen atoms were inserted into the amorphous samples in the vicinity of the atoms that have been identified in the LDOS analysis. In 
(a)

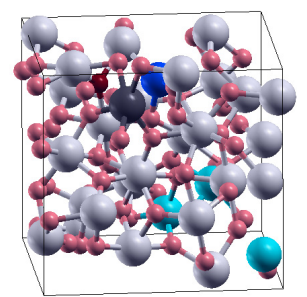

(c)

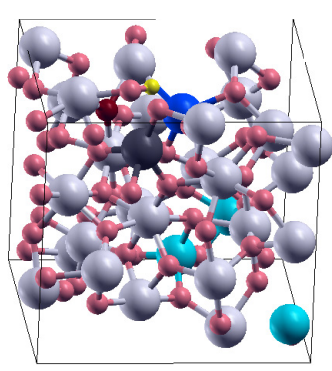

(b)

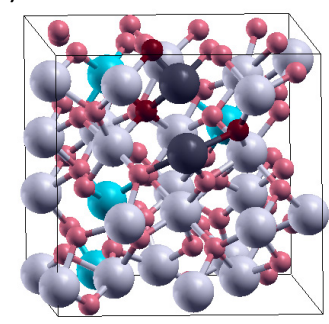

(d)

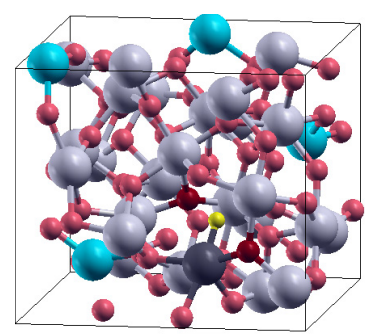

FIG. 8. Supercells containing a local oxygen deficiency and a metal-metal defect: (a) a-ZTO: $\mathrm{V}_{\ddot{\mathrm{O}}}$ sample s03, (b) a-ZTO: $\mathrm{V}_{\ddot{\mathrm{o}}}$ sample s02, (c) a-ZTO: $\mathrm{V}_{\ddot{\mathrm{o}}}+\mathrm{H}$ sample s03, and (d) a-ZTO: $\mathrm{V}_{\ddot{\mathrm{o}}}+\mathrm{H}$ sample s06. Sn, $\mathrm{Zn}, \mathrm{O}$, and $\mathrm{H}$ atoms are depicted as gray, blue, red, and yellow spheres, respectively. The $\mathrm{Sn}, \mathrm{Zn}$, and $\mathrm{O}$ atoms contributing most to the defect levels are highlighted by dark gray, dark blue, and dark red, respectively.

most cases, the defect level is shifted deeper into the band gap by approximately -0.2 to $-0.4 \mathrm{eV}$. The localization of the level onto a small number of atoms remains or even gets enhanced by the addition of hydrogen, while the $\mathrm{H}$ atom itself is never found to contribute directly to these defect levels. Note that for two samples the addition of hydrogen resulted in the elimination of the level after structural optimization. An interesting result was found for sample s06 of ZTO: $\mathrm{V}_{\ddot{\mathrm{O}}}$. Here, the local oxygen deficiency alone did not result in a deep level. However, when a $\mathrm{H}$ atom was added in close vicinity of the respective atomic environment, a deep level appeared with a strong contribution of the $\mathrm{H}$ atom, which is found to form a $\mathrm{Sn}-\mathrm{H}$ bond with the previously undercoordinated $\mathrm{Sn}$ atom [Fig. 8(d)].

The effect of hydrogen on the annealed stoichiometric samples was also investigated. Here, no deep levels were generated, and the DOS were found to be very similar to those shown in Figs. 6(c) and 6(d).

\section{CORRELATION OF DFT MODELS WITH EXPERIMENTAL RESULTS}

For annealing temperatures $<300^{\circ} \mathrm{C}$, the increase in $N_{e}$ occurs together with an increase in $\mu$ and is independent of the annealing atmosphere, as seen in Fig. 1. In this temperature range, the amorphous network does not appear to interact significantly with the chemical species coming from the annealing atmosphere and the changes are induced mostly by the temperature alone. Similar effects have been

described in literature and their origin is still under debate. For example, the $\mu$ and $N_{e}$ increase in zinc tin oxide [for layers with composition $\mathrm{Sn} /(\mathrm{Sn}+\mathrm{Zn})$ of 0.11 and 0.45 ] has been explained by a structural relaxation around $\mathrm{Sn}$ atoms, which may form $\mathrm{V}_{\ddot{\mathrm{O}}}$ donors instead of $\mathrm{V}_{\mathrm{O}}^{\mathrm{x}}$ [31]. Moreover, structural relaxations could result in the activation of hydrogen dopants [58] or charged metal ions [68] that act as dopants in a-ZTO. Furthermore, low temperature annealing could cause the reduction of nondominating scattering defects such as neutral impurities [59] or electron traps [20], which contribute to increase the mobility. Overall, it should be mentioned that these thermally induced structural relaxation processes are not significant, as confirmed by the TEM nanobeam diffraction experiments, which assess that both short- and medium-range order do not appear to change significantly with temperature (Fig. 4). In addition, the effect of the thermally induced local structural changes on the defect states, e.g., originating from undercoordinated oxygen, modestly increases with increasing temperature. As an example, only a modest reduction of defect states can be observed for samples annealed under nitrogen atmosphere at $500^{\circ} \mathrm{C}$ [Fig. 3(b)].

In contrast, a clear reduction in the density of subgap defects is observed when annealing in air at temperatures $>300^{\circ} \mathrm{C}$ [Figs. 1 and 2]. The electron mobility also increases significantly when annealing in air at these temperatures (up to $35 \mathrm{~cm}^{2} \mathrm{~V}^{-1} \mathrm{~s}^{-1}$, Fig. 3). The RBS data (cf. Table II) and the changes in optoelectronic properties when annealing in oxygen-rich atmospheres [Figs. 1-3] indicate that this effect mainly originates from the introduction of oxygen in the films. A reduction of the number of subgap defects with increased oxygen content, as observed when annealing in air, is in agreement with the atomistic DFT simulations. The source of subgap states in the upper half of the band gap are local oxygen deficiencies, which lead to undercoordinated $\mathrm{Sn}$ or $\mathrm{Zn}$ atoms [Fig. 7]. These oxygen-deficient states are known to act as donors $[4,5,37,69]$. At $T>300^{\circ} \mathrm{C}$, molecular oxygen from the atmosphere absorbs, dissociates, and eventually passivates these metallic dangling bonds, hence reducing the number of subgap defects and as a result $N_{e}$. Indeed, the decrease in $N_{e}$ with an increased oxygen concentration in the film can be explained by $\frac{1}{2} \mathrm{O}_{2}(g)+\mathrm{V}_{\ddot{\mathrm{O}}}+2 \mathrm{e}^{-} \leftrightarrow \mathrm{O}_{\mathrm{O}}^{\mathrm{x}}$.

In other words, the formation of $\mathrm{O}_{\mathrm{O}}^{\mathrm{x}}$ (or passivation of $\mathrm{V}_{\ddot{\mathrm{O}}}$ ) comes at the expense of charge carriers. While these defects act as dopants, they are also scattering centers, limiting the mobility. By reducing the number of such charge defects by annealing in air at high temperature, $\mu$ increases by $70 \%$ with respect to its original value, as shown in Fig. 1. Alternatively, several effects can explain the higher absorption of a-ZTO films annealed at high temperature in hydrogen [Fig. 3] , as well as the drastic increase in $N_{e}$ (see Fig. 1). First, the energy shift of subgap states $(-0.2$ to $-0.4 \mathrm{eV})$ in the band gap observed by the DFT calculations when introducing hydrogen near oxygen deficiencies corresponds to the measured broadening of the absorption energy range seen in Fig. 3. Second, the addition of hydrogen, as also inferred by DFT, might introduce new defect levels within the band gap. These defects may act as donors. And third, hydrogen may also create more oxygen deficiencies by removing oxygen from the sample close to the surface, which also increases subgap absorption as the $\mathrm{V}_{\ddot{\mathrm{O}}}$ density increases. Note that the number of samples studied 
by DFT does not allow for a predictive statistical analysis. A detailed theoretical study on the role of the metal-hydrogen complex, which includes the comparison of different DFT methods is in preparation.

While hydrogen allows an improved conductivity up to $577 \mathrm{~S} \mathrm{~cm}^{-1}$ by increasing $N_{e}$, it also results in more absorbing films. On the other hand, a remarkable electron mobility of $35 \mathrm{~cm}^{2} \mathrm{~V}^{-1} \mathrm{~s}^{-1}$ for a full amorphous indium-free electrode has been obtained by high-temperature annealing in oxygen-rich atmospheres. This results in the passivation of defects, specifically, undercoordinated $\mathrm{Sn}$ and $\mathrm{Zn}$ bonds, by introducing atomic oxygen to the amorphous network. This allows the formation of $\mathrm{Sn}-\mathrm{O}-\mathrm{Sn}$ ( or $\mathrm{Zn}-\mathrm{O}-\mathrm{Zn}, \mathrm{Zn}-\mathrm{O}-\mathrm{Sn}$ ) bonds, reducing the density of sub-band-gap defects (and hence the number of scattering centers), and with it reaching excellent optical properties with a conductivity of $445 \mathrm{~S} \mathrm{~cm}^{-1}$.

\section{CONCLUSION}

Using a combination of experimental techniques and atomistic calculations, the relation between sub-bandgap states and optoelectronic properties was clarified for sputtered a-ZTO thin films. The effects of temperature and gas atmosphere on the optoelectronic properties of the films were investigated. Annealing at low temperatures $\left(T \leqslant 300^{\circ} \mathrm{C}\right)$ induced a slight increase in $\mu$ and $N_{e}$, independently of the annealing atmosphere. These changes occur presumably due to either slight structural relaxations of the atomic network or the activation of hydrogen dopants with temperature.

At higher temperatures $\left(300^{\circ} \mathrm{C}<T \leqslant 500{ }^{\circ} \mathrm{C}\right)$, the optoelectronic properties of the films were strongly modified, with the final effect on $\mu$ and $N_{e}$ depending on the annealing atmosphere. Indeed, air treatments resulted in films with a low absorptance and a high mobility but a low free carrier concentration, while hydrogen-annealed films have high free carrier density but lower mobility and high absorptance.

As the films remained amorphous and free of nanoscopic voids in all cases, these results are explained by the generation or passivation of point defects. Indeed, DFT simulations identified local oxygen deficiencies and resulting metal atom clusters as the source of energy levels in the upper half of the band gap. These states act as the source of free carriers but also as scattering centers, hence limiting the mobility and deteriorating the optical properties. These defects are present in the film after deposition and can be modulated at high temperatures $\left(>300^{\circ} \mathrm{C}\right)$ by the annealing atmosphere. The strong increase in both free carrier concentration and absorption within the band gap when annealing in $\mathrm{H}_{2}$ is linked to both the formation of additional metal-hydrogen states within the band gap and the shift of the existing oxygen deficiency levels deeper into the band gap. On the other hand, the introduction of oxygen into the amorphous network during annealing in air at $500{ }^{\circ} \mathrm{C}$ passivates the oxygen deficiencies and results in the films with the best optoelectronic properties ( $\mu$ of $35 \mathrm{~cm}^{2} \mathrm{~V}^{-1} \mathrm{~s}^{-1}$ when compared to $21 \mathrm{~cm}^{2} \mathrm{~V}^{-1} \mathrm{~s}^{-1}$ after deposition).

Overall, these results indicate that a fine control of the oxygen and hydrogen contents is essential to optimize the optoelectronic properties of a-ZTO. Several strategies may be envisaged to further improve the properties of these films, e.g., by depositing other oxides together with a-ZTO to passivate these oxygen deficiencies in a controlled manner.

Considering that the a-ZTO films presented here are free of macroscopic defects, the tradeoff between optoelectronic properties suggests that a carrier transport optimum, i.e., a maximum mobility, has been reached for zinc tin oxide with this specific composition.

\section{ACKNOWLEDGMENTS}

This work is funded by the Swiss National Science Foundation Disco (Project No. CRSII2_154474), the European Union 7th Framework Program (Grant Agreement No. 312483ESTEEM2-I3), and the Czech Science Foundation (Project No. 16-10429J). The authors acknowledge Dr. Max Doebeli for the RBS measurements and Miglè Grauzinyte and Prof. Stefan Goedecker for fruitful discussion.
[1] A. Shah, Thin-Film Silicon Solar Cells (EPFL Press, Lausanne, Switzerland, 2010).

[2] J. Tauc, Mater. Res. Bull. 3, 37 (1968).

[3] S. Calnan and A. N. Tiwari, Thin Solid Films 518, 1839 (2010).

[4] A. Klein, J. Am. Ceram. Soc. 96, 331 (2013).

[5] T. Koida, H. Shibata, M. Kondo, K. Tsutsumi, A. Sakaguchi, and M. Suzuki, J. Appl. Phys. 111, 1 (2012).

[6] J. Robertson, K. Xiong, and S. J. Clark, Thin Solid Films 496, 1 (2006).

[7] M. Bazzani, A. Neroni, A. Calzolari, and A. Catellani, Appl. Phys. Lett. 98, 121907 (2011).

[8] W. Körner, P. Gumbsch, and C. Elsässer, Phys. Rev. B 86, 165210 (2012).

[9] W. Körner, D. F. Urban, D. M. Ramo, P. D. Bristowe, and C. Elsässer, Phys. Rev. B 90, 195142 (2014).

[10] W. Körner, D. F. Urban, and C. Elsässer, Phys. Status Solidi 6, 1 (2015).
[11] W. Körner and C. Elsässer, Thin Solid Films 555, 81 (2014).

[12] F. A. Kröger and H. J. Vink, J. Phys. Chem. Solids 5, 208 (1958).

[13] M. K. Jayaraj, K. J. Saji, K. Nomura, T. Kamiya, and H. Hosono, J. Vac. Sci. Technol. B 26, 495 (2008).

[14] T. Kamiya, K. Nomura, M. Hirano, and H. Hosono, Phys. Status Solidi C 5, 3098 (2008).

[15] K. Nomura, T. Kamiya, H. Yanagi, E. Ikenaga, K. Yang, K. Kobayashi, M. Hirano, and H. Hosono, Appl. Phys. Lett. 92, 202117 (2008).

[16] P. D. C. King, R. L. Lichti, Y. G. Celebi, J. M. Gil, R. C. Vilão, H. V. Alberto, J. Piroto Duarte, D. J. Payne, R. G. Egdell, I. McKenzie, C. F. McConville, S. F. J. Cox, and T. D. Veal, Phys. Rev. B 80, 081201(R) (2009).

[17] S. Limpijumnong, P. Reunchan, A. Janotti, and C. G. Van De Walle, Phys. Rev. B 80, 193202 (2009).

[18] F. Bekisli, M. Stavola, W. B. Fowler, L. Boatner, E. Spahr, and G. Lüpke, Phys. Rev. B 84, 035213 (2011). 
[19] W. M. Hlaing Oo, S. Tabatabaei, M. D. McCluskey, J. B. Varley, A. Janotti, and C. G. Van de Walle, Phys. Rev. B 82, 193201 (2010).

[20] Y. Hanyu, K. Domen, K. Nomura, H. Hiramatsu, H. Kumomi, H. Hosono, and T. Kamiya, Appl. Phys. Lett. 103, 202114 (2013).

[21] H. Tang, K. Ishikawa, K. Ide, H. Hiramatsu, S. Ueda, N. Ohashi, H. Kumomi, H. Hosono, and T. Kamiya, J. Appl. Phys. 118, 205703 (2015).

[22] A. K. Singh, A. Janotti, M. Scheffler, and C. G. Van de Walle, Phys. Rev. Lett. 101, 055502 (2008).

[23] C. G. Van de Walle, Phys. Rev. Lett. 85, 1012 (2000).

[24] J. Jiang, Y. Lu, B. K. Meyer, D. M. Hofmann, and M. Eickhoff, J. Appl. Phys. 119, 245703 (2016).

[25] D. M. Hofmann, A. Hofstaetter, F. Leiter, H. Zhou, F. Henecker, B. K. Meyer, S. B. Orlinskii, J. Schmidt, and P. G. Baranov, Phys. Rev. Lett. 88, 045504 (2002).

[26] Y. Mizokawa and S. Nakamura, Jpn. J. Appl. Phys. 13, 253 (1974).

[27] K. Ellmer, Nat. Photonics 6, 808 (2012).

[28] M. Morales-Masis, S. De Wolf, R. Woods-Robinson, J. W. Ager, and C. Ballif, Adv. Electron. Mater. 1600529 (2017).

[29] J. H. Ko, I. H. Kim, D. Kim, K. S. Lee, T. S. Lee, B. Cheong, and W. M. Kim, Appl. Surf. Sci. 253, 7398 (2007).

[30] R. A. Mereu, A. Le Donne, S. Trabattoni, M. Acciarri, and S. Binetti, J. Alloys Compd. 626, 112 (2015).

[31] Q. Zhu, Q. Ma, D. B. Buchholz, R. P. H. Chang, M. J. Bedzyk, and T. O. Mason, J. Appl. Phys. 115, 033512 (2014).

[32] T. Minami, J. Vac. Sci. Technol., A 17, 1765 (1999).

[33] H. Frenzel, D. Tobias, P. Schlupp, H. Von Wenckstern, and M. Grundmann, Phys. Status Solidi 1486, 1482 (2015).

[34] M. Morales-Masis, L. Ding, F. Dauzou, Q. Jeangros, A. HesslerWyser, S. Nicolay, and C. Ballif, APL Mater. 2, 096113 (2014).

[35] M. Morales-Masis, F. Dauzou, Q. Jeangros, A. Dabirian, H. Lifka, R. Gierth, M. Ruske, D. Moet, A. Hessler-Wyser, and C. Ballif, Adv. Funct. Mater. 26, 384 (2016).

[36] J. Werner, A. Walter, E. Rucavado, S.-J. Moon, D. Sacchetto, M. Rienaecker, R. Peibst, R. Brendel, X. Niquille, S. De Wolf, P. Löper, M. Morales-Masis, S. Nicolay, B. Niesen, and C. Ballif, Appl. Phys. Lett. 109, 233902 (2016).

[37] P. D. C. King and T. D. Veal, J. Phys.: Condens. Matter 23, 334214 (2011).

[38] S. Sallis, K. T. Butler, N. F. Quackenbush, D. S. Williams, M. Junda, D. A. Fischer, J. C. Woicik, N. J. Podraza, B. E. White, A. Walsh, and L. F. J. Piper, Appl. Phys. Lett. 104, 2012 (2014).

[39] F. Dauzou, P. C. P. Bouten, A. Dabirian, Y. Leterrier, C. Ballif, and M. Morales-Masis, Org. Electron. 35, 136 (2016).

[40] A. C. Boccara, D. Fournier, and J. Badoz, Appl. Phys. Lett. 36, 130 (1980).

[41] P. M. Voyles and D. A. Muller, Ultramicroscopy 93, 147 (2002).

[42] See Supplemental Material at http://link.aps.org/supplemental/ 10.1103/PhysRevB.95.245204 for information regarding electron microscopy techniques, temperature-dependent Hall effect, and FTIR fitting of Drude model.
[43] J. Gale, J. Chem. Soc., Faraday Trans. 93, 629 (1997).

[44] P. E. Blöchl, Phys. Rev. B 50, 17953 (1994).

[45] G. Kresse and J. Furthmüller, Phys. Rev. B 54, 11169 (1996).

[46] G. Kresse and D. Joubert, Phys. Rev. B 59, 1758 (1999).

[47] C. Elsasser, N. Takeuchi, K. M. Ho, C. T. Chan, P. Braun, and M. Fahnle, J. Phys.: Condens. Matter 2, 4371 (1990).

[48] K. M. Ho, C. Elsasser, C. T. Chan, and M. Fahnle, J. Phys.: Condens. Matter 4, 5189 (1992).

[49] B. Meyer, K. Hummler, C. Elsasser, and M. Fahnle, J. Phys.: Condens. Matter 7, 9201 (1995).

[50] F. Lechermann, M. Fähnle, B. Meyer, and C. Elsässer, Phys. Rev. B 69, 165116 (2004).

[51] D. Vogel, P. Krüger, and J. Pollmann, Phys. Rev. B 54, 5495 (1996).

[52] W. Körner and C. Elsässer, Phys. Rev. B 81, 085324 (2010).

[53] K. H. Ko, H. S. So, D. H. Jung, J. W. Park, and H. Lee, J. Appl. Phys. 119, 135302 (2016).

[54] K. L. Chopra, S. Major, and D. K. Pandya, Thin Solid Films 102, 1 (1983).

[55] M. Morales-Masis, S. Martin De Nicolas, J. Holovsky, S. De Wolf, and C. Ballif, IEEE J. Photovoltaics 5, 1340 (2015).

[56] M. N. Mullings, C. Hägglund, J. T. Tanskanen, Y. Yee, S. Geyer, and S. F. Bent, Thin Solid Films 556, 186 (2014).

[57] K. Nomura, T. Kamiya, and H. Hosono, ECS J. Solid State Sci. Technol. 2, P5 (2013).

[58] A. Janotti and C. G. Van de Walle, Nat. Mater. 6, 44 (2007).

[59] N. Ito, Y. Sato, P. K. Song, A. Kaijio, K. Inoue, and Y. Shigesato, Thin Solid Films 496, 99 (2006).

[60] B. Macco, H. C. M. Knoops, and W. M. M. Kessels, ACS Appl. Mater. Interfaces 7, 16723 (2015).

[61] B. Stjerna, E. Olsson, and C. G. Granqvist, J. Appl. Phys. 76, 3797 (1994).

[62] R. B. Dingle, London, Edinburgh, Dublin Philos. Mag. J. Sci. 46, 831 (1955).

[63] B. Kamp, R. Merkle, R. Lauck, and J. Maier, J. Solid State Chem. 178, 3027 (2005).

[64] J. I. Pankove, Optical Processes in Semiconductors (Courier Corporation, North Chelmsford, MA, 2012).

[65] W. Körner, D. F. Urban, and C. Elsässer, J. Appl. Phys. 114, 163704 (2013).

[66] W. Körner, D. F. Urban, and C. Elsässer, Phys. Status Solidi 212, 1476 (2015).

[67] M. J. Wahila, K. T. Butler, Z. W. Lebens-Higgins, C. H. Hendon, A. S. Nandur, R. E. Treharne, N. F. Quackenbush, S. Sallis, K. Mason, H. Paik, D. G. Schlom, J. C. Woicik, J. Guo, D. A. Arena, B. E. White, G. W. Watson, A. Walsh, and L. F. J. Piper, Chem. Mater. 28, 4706 (2016).

[68] I. H. Kim, J. H. Ko, D. Kim, K. S. Lee, T. S. Lee, J.-H. Jeong, B. Cheong, Y.-J. Baik, and W. M. Kim, Thin Solid Films 515, 2475 (2006).

[69] S. Narushima, M. Orita, M. Hirano, and H. Hosono, Phys. Rev. B 66, 035203 (2002). 